\title{
Doppler-Shifted Inertial Oscillations on a $\beta$ Plane
}

\author{
Xiaoming Zhai, Richard J. Greatbatch, and Jinyu Sheng \\ Department of Oceanography, Dalhousie University, Halifax, Nova Scotia, Canada
}

(Manuscript received 12 August 2004, in final form 31 August 2004)

\begin{abstract}
On the spherical earth, and in the absence of a background flow, the poleward propagation of nearinertial oscillations is restricted by the turning latitude. A background flow, on the other hand, provides a way to increase the apparent frequency of near-inertial waves through Doppler shifting. In this note, it is shown that near-inertial oscillations can be advected to latitudes higher than their turning latitude. Associated with the poleward advection there is a squeezing of the meridional wavelength. A numerical model is used to verify this result. The squeezed inertial oscillations are vulnerable to nonlinear interactions, which could eventually lead to small-scale dissipation and mixing.
\end{abstract}

\section{Introduction}

It is well known that there is an asymmetry in the meridional propagation of near-inertial waves since waves that propagate poleward soon reach their turning latitude and are reflected back toward the equator (Geisler and Dickinson 1972; Anderson and Gill 1979; Gill 1984; Garrett 2001). The theory is supported by observations (e.g., Fu 1981; Chiswell 2003; Alford 2003). Furthermore, the equatorward propagation of near-inertial waves is important for the redistribution of the energy available for ocean mixing (Alford 2003). Near-inertial waves can also interact with background currents and mesoscale eddies during their propagation (Olbers 1981; D'Asaro 1995; Lee and Eriksen 1997). Kunze (1985) showed that, for near-inertial waves propagating in geostrophic shear, horizontally nonuniform relative vorticity has the same effect as the variation of the Coriolis parameter with latitude. As a consequence, near-inertial energy can be trapped in regions of anticyclonic relative vorticity. In addition, White (1972) found evidence from mooring data that a uniform background current can cause a Doppler shift of the inertial frequency. Further evidence of this effect

Corresponding author address: Xiaoming Zhai, Department of Oceanography, Dalhousie University, Halifax, NS B3H 4J1, Canada.

E-mail: xiaoming.zhai@phys.ocean.dal.ca has been provided by a case study of Doppler-shifted inertial oscillations in the Norwegian Coastal Current (Orvik and Mork 1995).

Zhai et al. (2004) recently studied the zonal advective spreading of storm-induced inertial oscillations in a model of the northwest Atlantic Ocean. The fact that inertial oscillations can be advected by a background flow raises the question of what happens if inertial oscillations are advected poleward beyond their turning latitude, where they cannot exist by themselves since these waves are strictly subinertial. Doppler shifting, on the other hand, provides a way to increase the apparent wave frequency. In this note we show that it is possible for near-inertial energy to be carried poleward because of Doppler shifting and we provide a simple theory to predict the change of shape of the inertial waves as they are advected poleward on a $\beta$ plane.

\section{Analytic model}

We start from the reduced-gravity model and then extend the theory to a continuously stratified ocean. The equations governing linear wave motion on an $f$ plane in the presence of a barotropic, uniform poleward flow are

$$
\begin{aligned}
u_{t}+V u_{y}-f v & =-g^{\prime} \eta_{x}, \\
v_{t}+V v_{y}+f u & =-g^{\prime} \eta_{y}, \quad \text { and } \\
\eta_{t}+V \eta_{y}+H\left(u_{x}+v_{y}\right) & =0,
\end{aligned}
$$




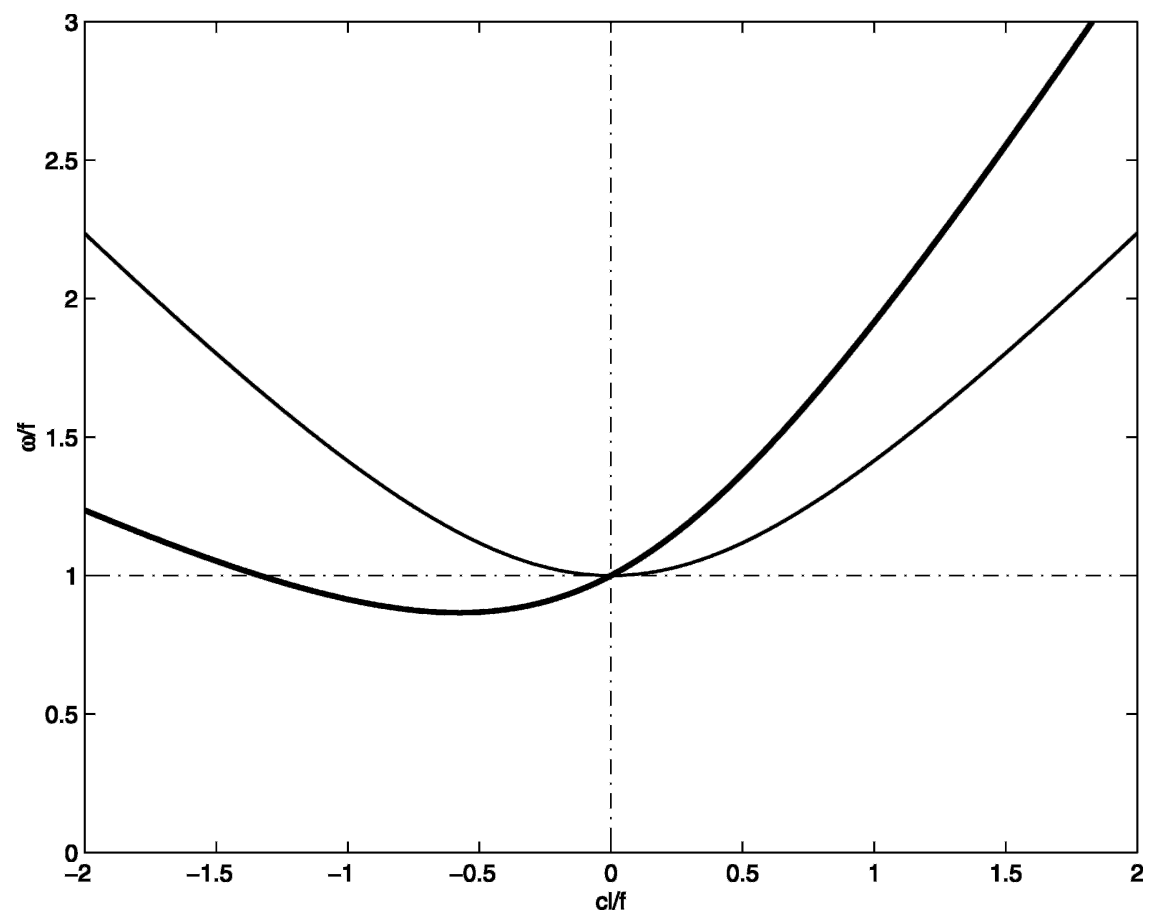

FIG. 1. The dispersion relationship modified by the Doppler shift due to a uniform poleward flow. The thin line represents wave frequency without background current, and the thick line represents wave frequency with background current. Here $V=0.5 \mathrm{~m} \mathrm{~s}^{-1}, c=\sqrt{g^{\prime} H}=1.0 \mathrm{~m}$ $\mathrm{s}^{-1}$, and the zonal wavenumber $k=0$.

where $(u, v)$ are perturbation velocities in $x$ and $y$ directions, respectively; $f$ is the Coriolis parameter; $V$ is the poleward background current; $H$ is the averaged upper-layer depth; $g^{\prime}$ is the reduced gravity [defined as $g\left(\rho_{2}-\rho_{1}\right) / \rho_{2}$, where $\rho_{1}$ and $\rho_{2}$ are upper- and lowerlayer densities, respectively]; and $\eta$ is the downward displacement of the interface between the two layers.

The divergence and vorticity equations are, respectively,

$$
\left(\frac{\partial}{\partial t}+V \frac{\partial}{\partial y}\right)\left(u_{x}+v_{y}\right)-f\left(v_{x}-u_{y}\right)=-g^{\prime}\left(\eta_{x x}+\eta_{y y}\right)
$$

and

$$
\left(\frac{\partial}{\partial t}+V \frac{\partial}{\partial y}\right)\left(v_{x}-u_{y}\right)+f\left(u_{x}-v_{y}\right)=0 .
$$

Combining Eqs. (3), (4), and (5) we get the equation for $\eta$ :

$$
\begin{array}{r}
{\left[\left(\frac{\partial}{\partial t}+V \frac{\partial}{\partial y}\right)^{2}+f^{2}\right]\left(\eta_{t}+V \eta_{y}\right)} \\
=\left(\frac{\partial}{\partial t}+V \frac{\partial}{\partial y}\right)\left[g^{\prime} H\left(\eta_{x x}+\eta_{y y}\right)\right] .
\end{array}
$$

Looking for solutions of the form

$$
\eta=\eta_{0} e^{i(k x+l y-\omega t)}
$$

where $(k, l)$ are the horizontal wavenumbers and $\omega$ is the frequency, leads to the dispersion relationship

$$
(\omega-V l)^{2}=f^{2}+g^{\prime} H\left(k^{2}+l^{2}\right)
$$

plotted in Fig. 1. We note that $\omega$ can be less than $f$ when there is a poleward flow in the Northern Hemisphere $(V>0$ and $l<0)$.

The theory can be easily extended to continuous stratification by noting, following Gill (1982), that the equations for a continuously stratified, flat-bottomed ocean can be separated into an infinite set of vertical normal modes. For each baroclinic mode, the equations are the same as Eqs. (1), (2), and (3) but with $g^{\prime}$ replaced by $g$ and a different $H$ (equivalent depth) for each mode. For a uniform stratification (i.e., the buoyancy frequency, $N$, independent of depth) the dispersion relationship corresponding to (8) takes the form

$$
(\omega-V l)^{2}=f^{2}+\frac{N^{2}\left(k^{2}+l^{2}\right)}{m^{2}},
$$

where $m$ is the vertical wavenumber (for the $n$th baroclinic normal mode, $m=n \pi / H$, where $n \geq 1$ ). When 


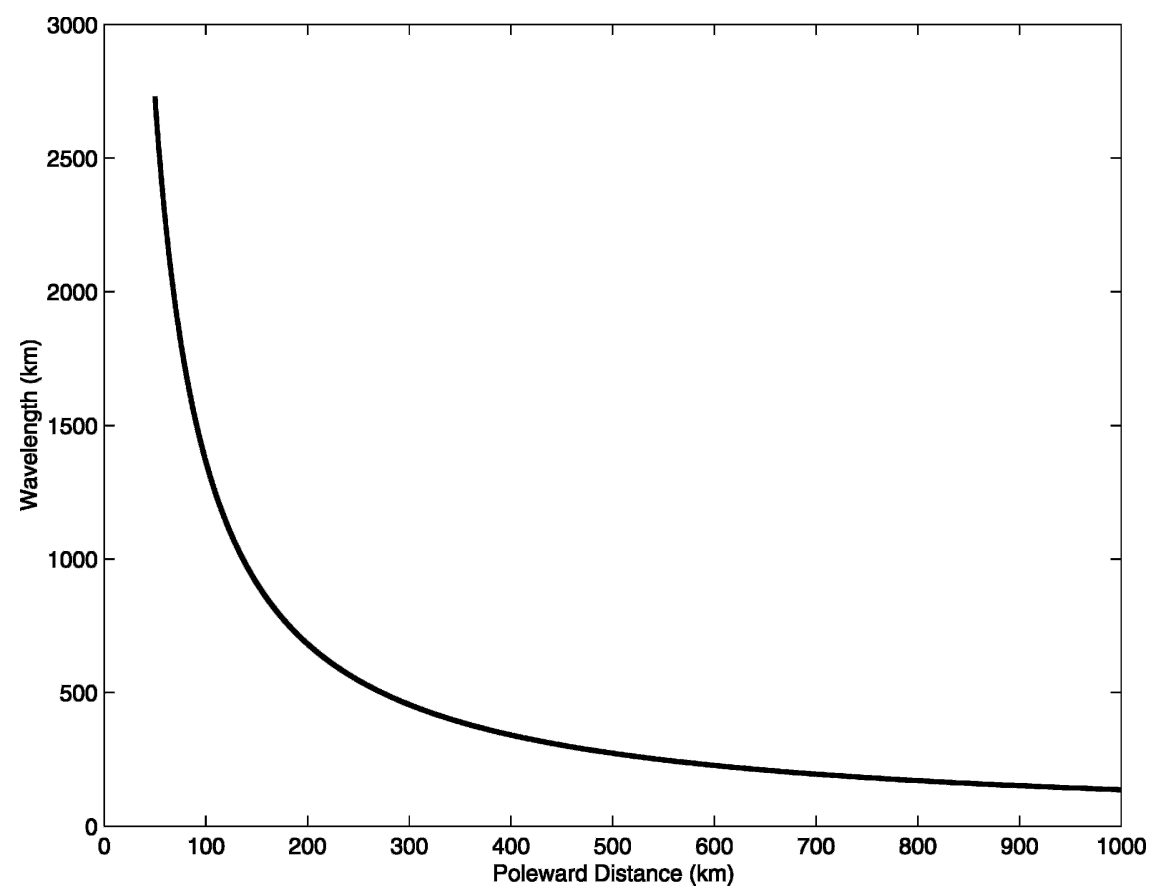

FIG. 2. Variation of near-inertial wavelength against the poleward distance from the generation latitude in the diagnostic case. For this plot $\beta=2.3 \times 10^{-11} \mathrm{~m}^{-1} \mathrm{~s}^{-1}$ and $V=0.5 \mathrm{~m} \mathrm{~s}^{-1}$.

$V=0$ (no background flow), Eq. (9) reduces to Eq. (8.4.23) in Gill (1982).

\section{a. On a $\beta$ plane}

We first investigate this problem in the "diagnostic" case. Diagnostic means that the density field is specified and the horizontal pressure gradients are no longer interactive with the flow so that baroclinic dispersion of inertial-gravity waves is excluded. ${ }^{1}$ On an $f$ plane, the dispersion relation is then the same as in Eqs. (8) or (9) but with the terms involving $g^{\prime}$ and $N$ neglected [i.e., $(\omega$ $\left.-V l)^{2}=f^{2}\right]$ and is the same for both the reduced gravity and continuously stratified models. To take account of the variation of the Coriolis parameter with latitude, we make use of the Wentzel-Kramers-Brillouin-Jeffreys (WKBJ) approximation (see Gill 1982). The dispersion relationship can then be written as

$$
(\omega-V l)^{2}=\left(f_{0}+\beta y\right)^{2},
$$

where $f$ is replaced by $f_{0}+\beta y$ on a $\beta$ plane; $f_{0}$ is the local inertial frequency at the latitude where the nearinertial waves are generated, and $\beta$ is the variation of $f$ with latitude. The use of the WKBJ approximation to

\footnotetext{
${ }^{1}$ The diagnostic case is appropriate when the horizontal length scales are large in comparison with the internal radius of deformation; see Greatbatch (1983).
}

write Eq. (10) assumes that the wavelength implied by the meridional wavenumber, $l$, is small relative to the scale on which $f$ varies with latitude (i.e., the planetary scale). The same approach has been used by Anderson and Gill (1979) [cf. our Eq. (10) with their Eq. (8)] and Garrett [2001; see his Eq. (7)].

For near-inertial waves $\omega$ is very close to $f_{0}$, and Eq. (10) can be approximated by

$$
-V l=\beta y .
$$

It follows that the meridional wavenumber is given by $-\beta y / V$, where $y / V=t$ is the advective time scale from the generation latitude $y=0$. For a poleward background current, $V$ is positive, $y$ is positive, and $\beta$ is positive, and so $l$ is negative and its magnitude increases linearly with latitude during the advection. This indicates that the inertial oscillations shrink meridionally when carried poleward (Fig. 2). The same is true for inertial oscillations that are carried equatorward, as can be seen from Eq. (11), since this time both $V$ and $y$ are negative, and $l$ is once again negative.

The near-inertial energy is carried by the group velocity. The horizontal group velocity in the diagnostic case is

$$
\frac{\partial \omega}{\partial l}=V
$$

which means the near-inertial energy is transported poleward solely by the background current at the speed 
of the background current velocity and the near-inertial waves act as passive tracers. The vertical group velocity is $\partial \omega / \partial m=0$ in this case, which indicates that the nearinertial energy is trapped in the mixed layer and dissipated there, and this energy is not available for deep ocean mixing.

However, in the real ocean, the density field is free to interact with the flow. In this case, baroclinic dispersion can play a role and the near-inertial waves become active tracers. To illustrate this case, we assume vertically uniform stratification. As before, we use the WKBJ approximation to write Eq. (9) as

$$
(\omega-V l)^{2}=\left(f_{0}+\beta y\right)^{2}+\frac{N^{2} l^{2}}{m^{2}},
$$

where, for simplicity, only vertical and meridional propagation are considered. Assuming once again that $\omega \approx f_{0}$ it follows that $l<-\beta y / V$, which indicates that the inertial oscillations shrink quicker meridionally than in the diagnostic case. The horizontal group velocity is given by

$$
\frac{\partial \omega}{\partial l}=V+\frac{N^{2} l}{m^{2}(\omega-V l)},
$$

showing that the horizontal group velocity is determined by the sum of the background advective velocity, $V$, and a modified horizontal wave dispersion term. Since $l$ is negative, near-inertial energy is transported poleward at a speed less than the background current velocity. Similarly, the vertical group velocity is given by

$$
\frac{\partial \omega}{\partial m}=-\frac{N^{2} l^{2}}{m^{3}(\omega-V l)} .
$$

The negative sign indicates downward (upward) propagation of the near-inertial energy when phase velocity is upward (downward), as in the case when $V=0$ (see Gill 1982). Since $\omega-V l>\omega$, the amplitude of the vertical group velocity is reduced by a factor of $(\omega-$ $V l) / \omega$ from the case with $V=0$. For storm-generated near-inertial waves that are carried poleward by a background current, the downward propagation of the nearinertial energy is therefore reduced and less energy escapes from the mixed layer to the deep ocean.

Zhai et al. (2004) showed that inertial energy can be carried by a background current (in their case, the Gulf Stream) to remote regions in a model of the northwest Atlantic Ocean. The dominance of advection over wave dispersion in their model can easily be demonstrated using the theory developed here but modified to take account of advection by a zonal, rather than meridional flow. The zonal component of the horizontal group velocity is then

$$
\frac{\partial \omega}{\partial k}=U+\frac{N^{2} k}{m^{2}(\omega-V k)},
$$

where $U$ is the zonal background flow. For the size of storm in Zhai et al. (2004), $k$ is about $2 \times 10^{-5} \mathrm{~m}^{-1}, \omega$ is about $10^{-4} \mathrm{~s}^{-1}$, we take $N^{2} \mathrm{~m}^{-2}=1.0 \mathrm{~m}^{2} \mathrm{~s}^{-2}$, and the background current velocity $U$ is close to $1 \mathrm{~m} \mathrm{~s}^{-1}$. Thus

$$
\frac{\text { dispersive processes }}{\text { advective processes }}=\frac{N^{2} k}{m^{2}(\omega-V k) U}=\frac{0.2}{1},
$$

which indicates that advective processes dominate the near-inertial wave dispersion in their case.

\section{b. On an $f$ plane}

On the $f$ plane, the $\beta$ effect is excluded. In the diagnostic case, the dispersion relationship reduces to

$$
(\omega-V l)^{2}=f_{0}^{2}
$$

which shows that $V l=$ const. As long as the poleward background current $V$ is spatially uniform, the meridional wavenumber $l$ is constant, which indicates that the inertial oscillations keep their shape during the poleward advection.

When baroclinic wave dispersion plays a role, northward energy propagation is enhanced by the northward advection, while the southward energy propagation is reduced as seen from the dispersion curve (Fig. 1). Depending on the strength of the background flow, the first baroclinic mode is the mode that can most easily overcome the poleward advection and propagate equatorward (we shall see evidence of this in the model results presented below). This is because the quadratic dependence on horizontal wavenumber in Eq. (8) is stronger the larger the gravity wave speed $\sqrt{g^{\prime} H}$, and for the baroclinic modes the first mode has the largest wave speed. [Equivalently, among the baroclinic modes, the vertical wavenumber $m$ is smallest for the first baroclinic mode, from which it follows that the dispersion term on the right-hand side of Eq. (14) is largest for the first mode.]

\section{Numerical model}

The ocean model used here is the same as in Zhai et al. (2004), except that we use an idealized model setup. The model domain is rectangular and covers the area between $30^{\circ}$ and $60^{\circ} \mathrm{N}, 30^{\circ}$ and $60^{\circ} \mathrm{W}$ with two open boundaries at the south and the north and two solid boundaries at the east and the west. The horizontal 
resolution is about $20 \mathrm{~km}$ and there are 31 unevenly distributed vertical levels with the centers of the top five levels located at 5, 16, 29, 44, and $61 \mathrm{~m}$, respectively. The stratification is horizontally uniform, with a vertical temperature structure representative of the midlatitude Atlantic Ocean (Fig. 3). The salinity is set everywhere uniform. An initial poleward current of 50 $\mathrm{cm} \mathrm{s}^{-1}$ is introduced everywhere in the domain and maintained by the open boundaries throughout the simulation. To prevent western intensification of the barotropic background flow, the bottom relief is designed in such a way as to compensate for the variation of the Coriolis parameter with latitude. The water depth is a function of longitude and latitude and is given by

$$
H(x, y)=H(x) \times 2 \sin (\phi),
$$

where the zonal dependence $H(x)$ is a linear slope and $\phi$ is the latitude. In this way,

$$
\frac{f}{H}=\frac{2 \Omega \sin (\phi)}{H(x) \sin (\phi)}=\frac{\Omega}{H(x)},
$$

where $\Omega$ is the earth's rotation rate and the $f / H$ contours coincide with lines of longitude. The poleward background flow is then an almost spatially uniform flow throughout the model runs, following the $\mathrm{flH}$ contours rather than forming an intensified western boundary current as happens with a flat bottom. Storm forcing is specified following Chang and Anthes (1978) and is used to generate the inertial oscillations. The wind stress for the storm is

$$
\tau=\tau_{\max } \times\left\{\begin{array}{cc}
r / r_{\min } & 0 \leq r \leq r_{\min } \\
\left(r_{\max }-r\right) /\left(r_{\max }-r_{\min }\right) & r_{\min } \leq r \leq r_{\max }, \\
0 & r \geq r_{\max }
\end{array}\right.
$$

where $\tau$ is the amplitude of the tangential wind stress with respect to the storm center (the radial wind stress is set to zero), and $r$ is the radial distance from the center. Here, we put $r_{\min }=30 \mathrm{~km}, r_{\max }=300 \mathrm{~km}$, and $\tau_{\max }=3 \mathrm{~N} \mathrm{~m}^{-2}$ for a typical storm, the same as in Zhai et al. (2004). The storm track is specified to be zonal from $55^{\circ}$ to $35^{\circ} \mathrm{W}$ at $43^{\circ} \mathrm{N}$ latitude and the translation speed of the storm is $8.5 \mathrm{~m} \mathrm{~s}^{-1}$. Radiation open boundary conditions are used at the south and north boundaries. Since the boundaries are far away from the area of interest, they are small in their effect.

\section{Results}

a. On a $\beta$ plane

Two prognostic model runs are conducted on a $\beta$ plane: one with storm forcing and the other without

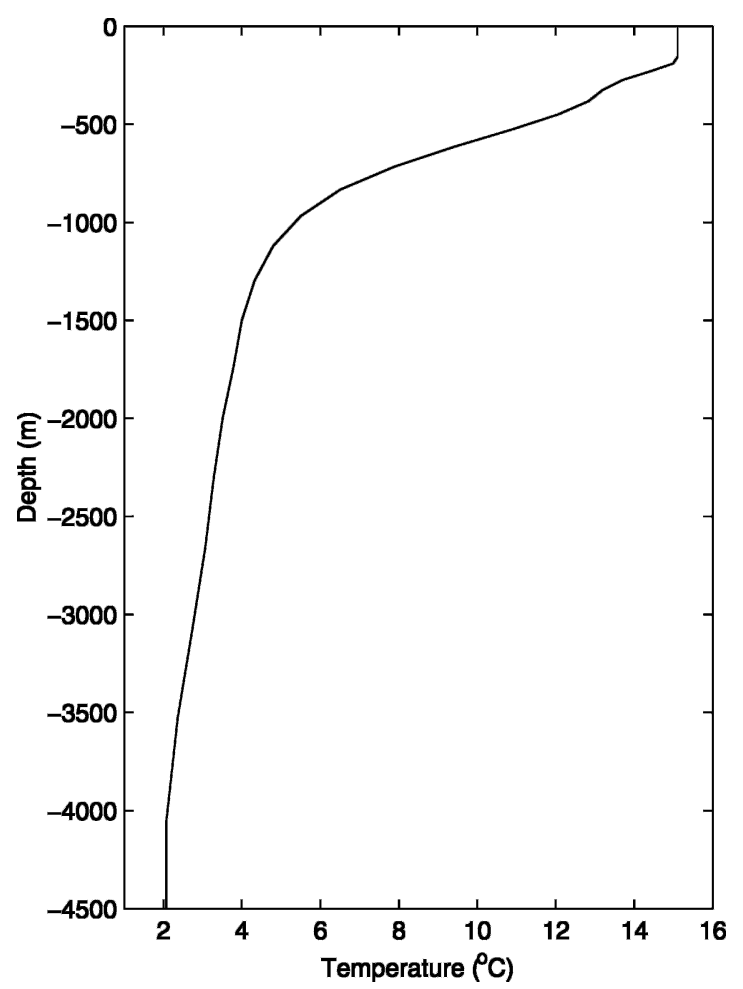

FIG. 3. Initial vertical temperature profile used in the model. Note that the temperature is initially horizontally uniform.

storm forcing. The velocity differences between the two model runs are used to represent the oceanic response to the storm forcing. To extract the near-inertial response, a bandpass filter centered at the local $\left(43^{\circ} \mathrm{N}\right)$ inertial frequency is used. The temporal and spatial evolution of the inertial-band filtered zonal currents at the sea surface is shown in Fig. 4. The near-inertial currents are initially biased to the right of the storm track (not shown), consistent with previous studies (e.g., Price 1981). They are gradually advected poleward and the inertial oscillations are almost centered at the storm track at day 4. As the inertial oscillations are carried further poleward, they are squeezed meridionally as predicted by the linear theory, while the zonal wavenumber is well preserved. The $\beta$-dispersion effect is also evident after day 7, indicated by the near-inertial waves propagating equatorward, but it seems that most energy is carried poleward of the storm track. A vertical transect along the middle longitude is shown in Fig. 5. This figure is similar to Fig. 12 in Gill (1984), but note that the source of the equatorward propagating waves is carried several hundred kilometers poleward of the storm track by the background flow. In addition, in contrast to the situation in Gill (1984) where the background flow is zero, the presence of the poleward background flow inhibits the equatorward dispersion of 


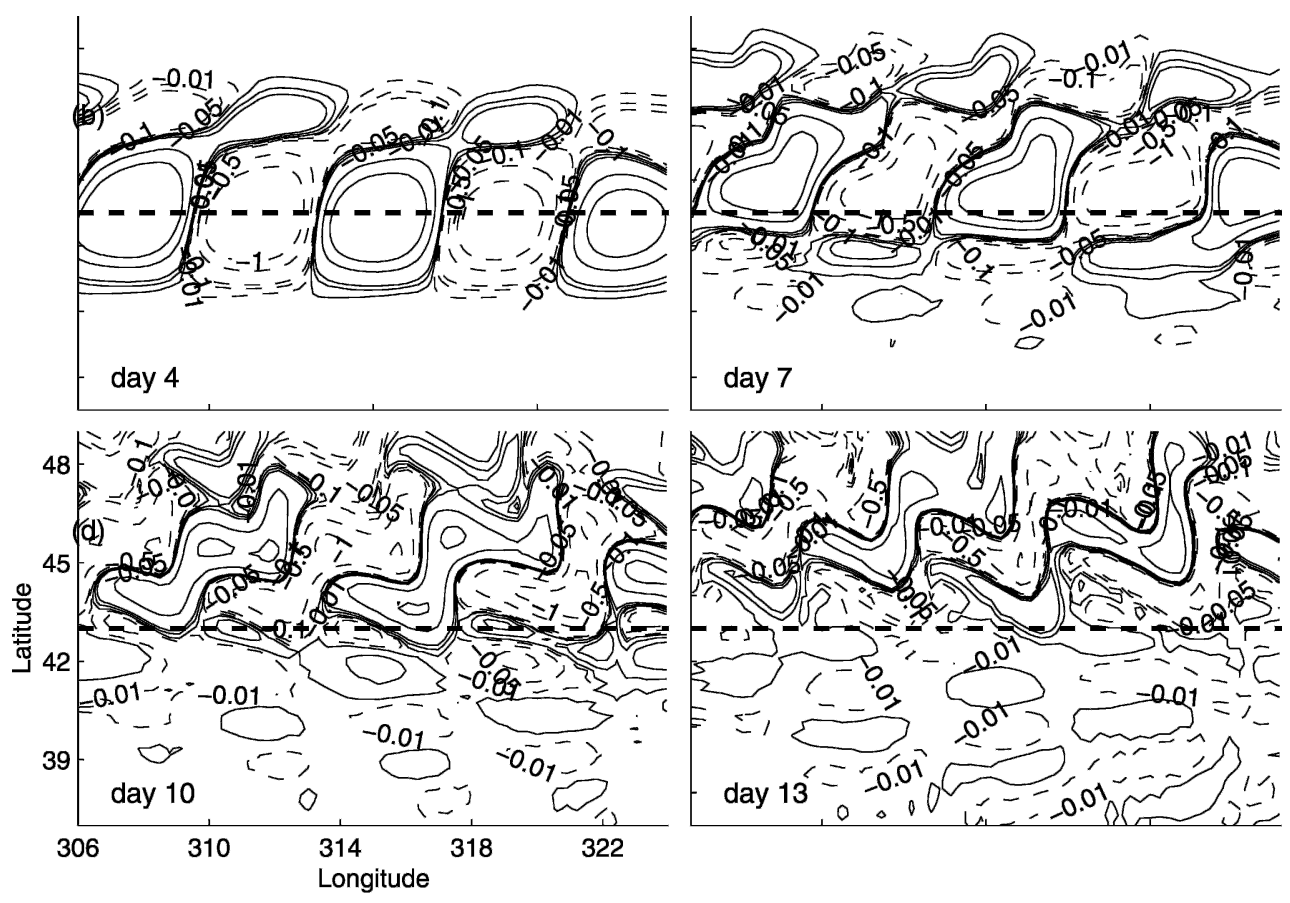

FIG. 4. Temporal evolution of the inertial-band filtered zonal current at the sea surface in the prognostic run on a $\beta$ plane (unit: $\mathrm{m} \mathrm{s}^{-1}$ ). The dashed line represents the storm track.

baroclinic modes higher than the first mode (see the end of section 2). In fact, it seems that only the first baroclinic mode can make its way equatorward, as indicated by the $180^{\circ}$ phase difference between the nearsurface and near-bottom currents equatorward of the storm track in Fig. 5.

Two additional diagnostic model runs (i.e., one with the storm forcing and one without) are conducted on the $\beta$ plane, with the density field specified from the initial condition, in which case the horizontal pressure gradients are independent of the model-calculated temperature so that the baroclinic dispersion of the inertial-gravity waves is excluded. This effect is evident in Fig. 6. There is no baroclinic dispersion and the nearinertial energy is confined in the mixed layer except for the (deep) inertial pumping that is also carried poleward of the storm track. The inertial oscillations act like passive tracers in the diagnostic case and the nearinertial energy occurs only poleward of the storm track at day 13 and is eventually dissipated there. For $t \approx 10$ days and $V \approx 50 \mathrm{~m} \mathrm{~s}^{-1}$, the advection distance is roughly about $430 \mathrm{~km}$, consistent with what is shown in Fig. 7. The meridional width of the inertial oscillations is about $300 \mathrm{~km}$ at day 13 after being advected poleward for about $500 \mathrm{~km}$, close to the analytical prediction in Fig. 2. The advection distance of the inertial oscillations in the prognostic run is a little shorter than that in the diagnostic run, because of the second term in
Eq. (14), which is negative and representing the wave propagation. The most revealing fact is that the diagnostic run captures the essential features of the prognostic run-that is, the poleward advection of the nearinertial energy and the meridional squeezing of the near-inertial oscillations (cf. Fig. 4 with Fig. 7).

\section{b. On an $f$ plane}

In the diagnostic run on the $f$ plane, the inertial oscillations act solely as passive tracers (Fig. 8). There is no squeezing of the meridional wavelength and the shape of the inertial oscillations are well preserved during the advection, which is consistent with the analytical solution. In the prognostic case on the $f$ plane, baroclinic dispersion takes effect and there is energy leakage both northward and southward through the propagation of the near-inertial waves (Fig. 9). This can be explained by the concepts of modal separation and model interference as described in Gill (1984) and Zervakis and Levine (1995). The inertial oscillations, though carried northward by the background current, do not change much in their shape in contrast to what happens on the $\beta$ plane.

\section{Summary and discussion}

Inertial oscillations can be carried poleward by a background flow beyond their turning latitude because 

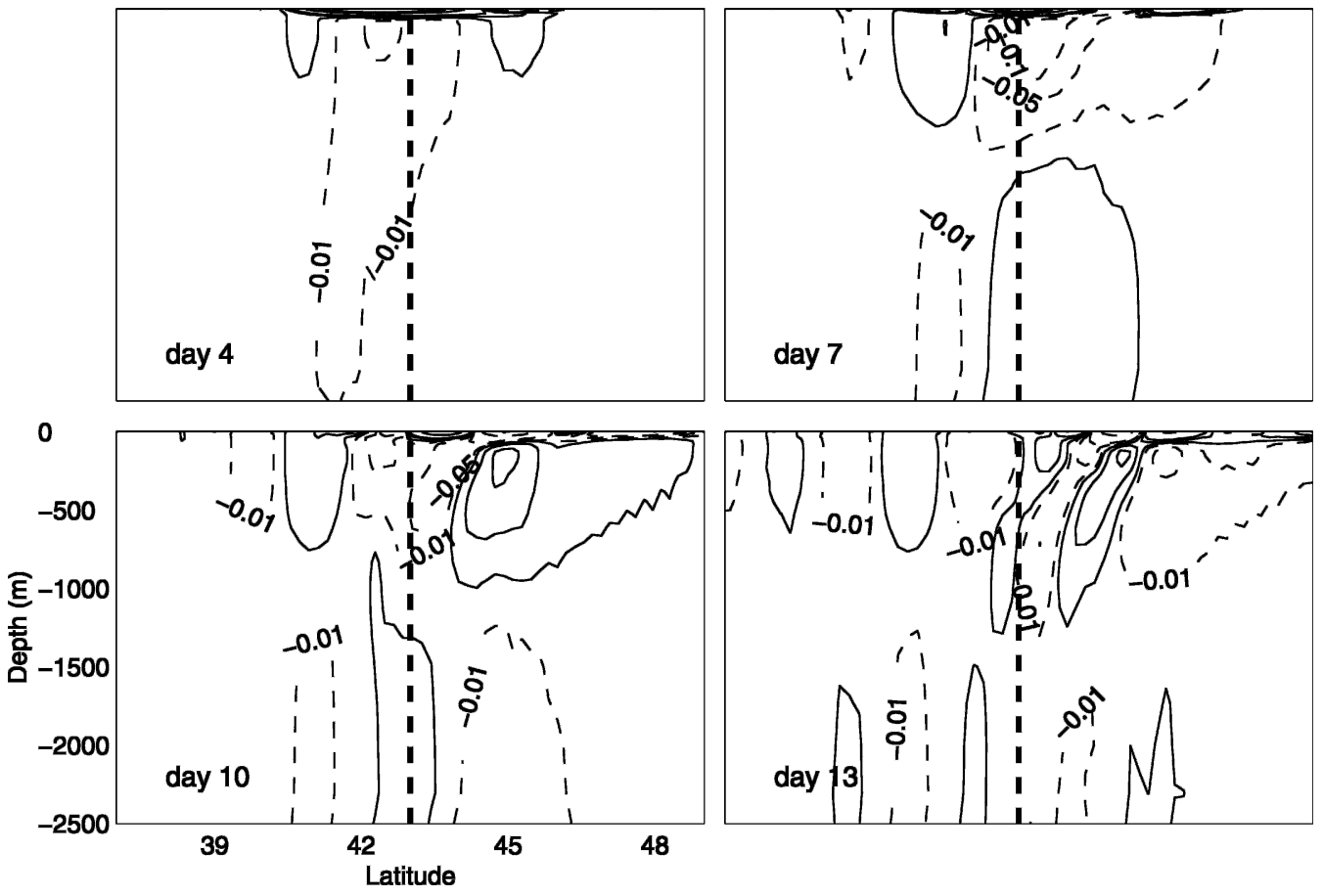

FIG. 5. Vertical transect showing the temporal evolution of the inertial-band filtered zonal current in the prognostic run on a $\beta$ plane (unit: $\mathrm{m} \mathrm{s}^{-1}$ ) in the upper $2500 \mathrm{~m}$. The dashed line shows where the storm center intersects the transect.
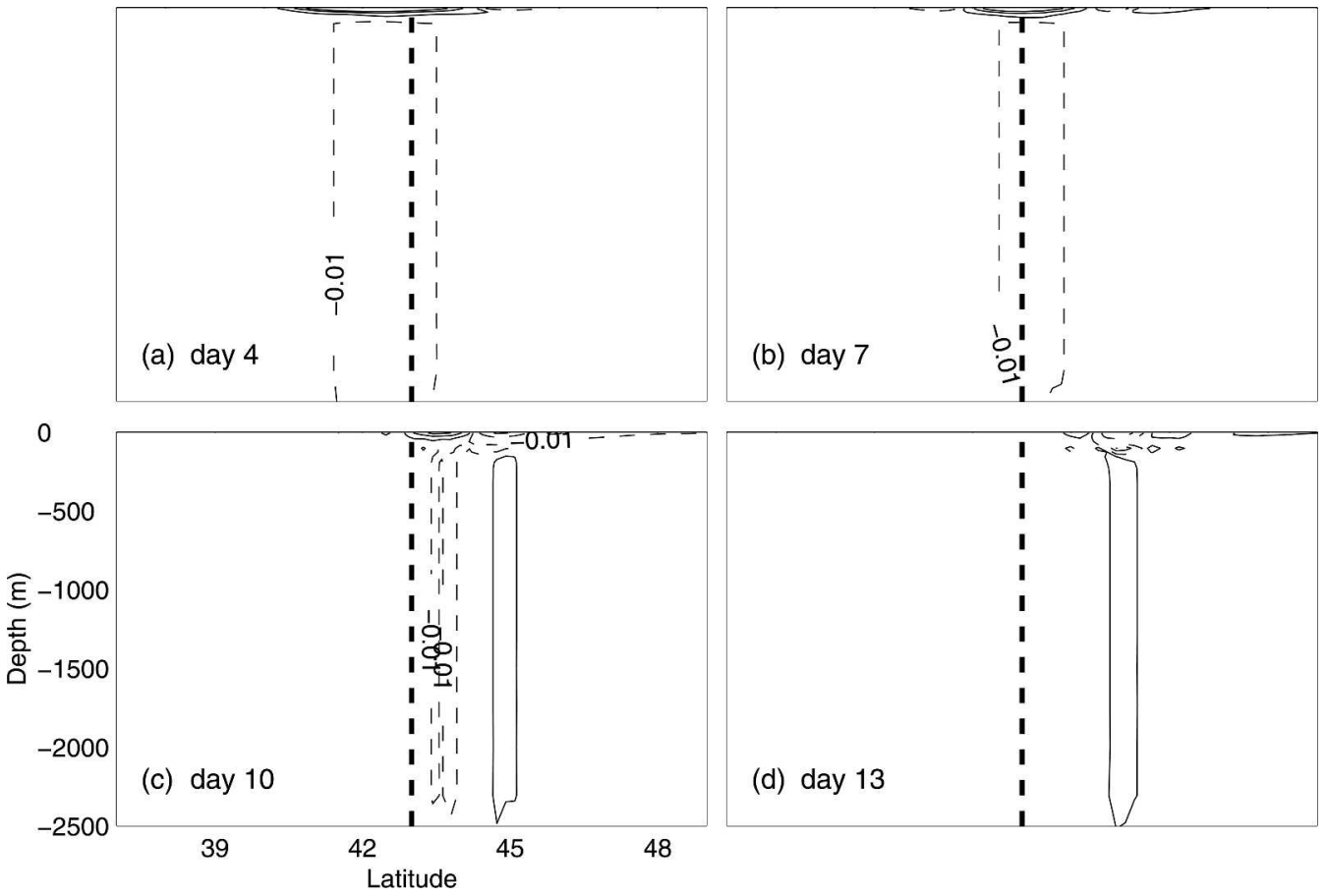

FIG. 6. Vertical transect showing the temporal evolution of the inertial-band filtered zonal current in the diagnostic run on a $\beta$ plane (unit: $\mathrm{m} \mathrm{s}^{-1}$ ) in the upper $2500 \mathrm{~m}$. The dashed line shows where the storm center intersects the transect. 


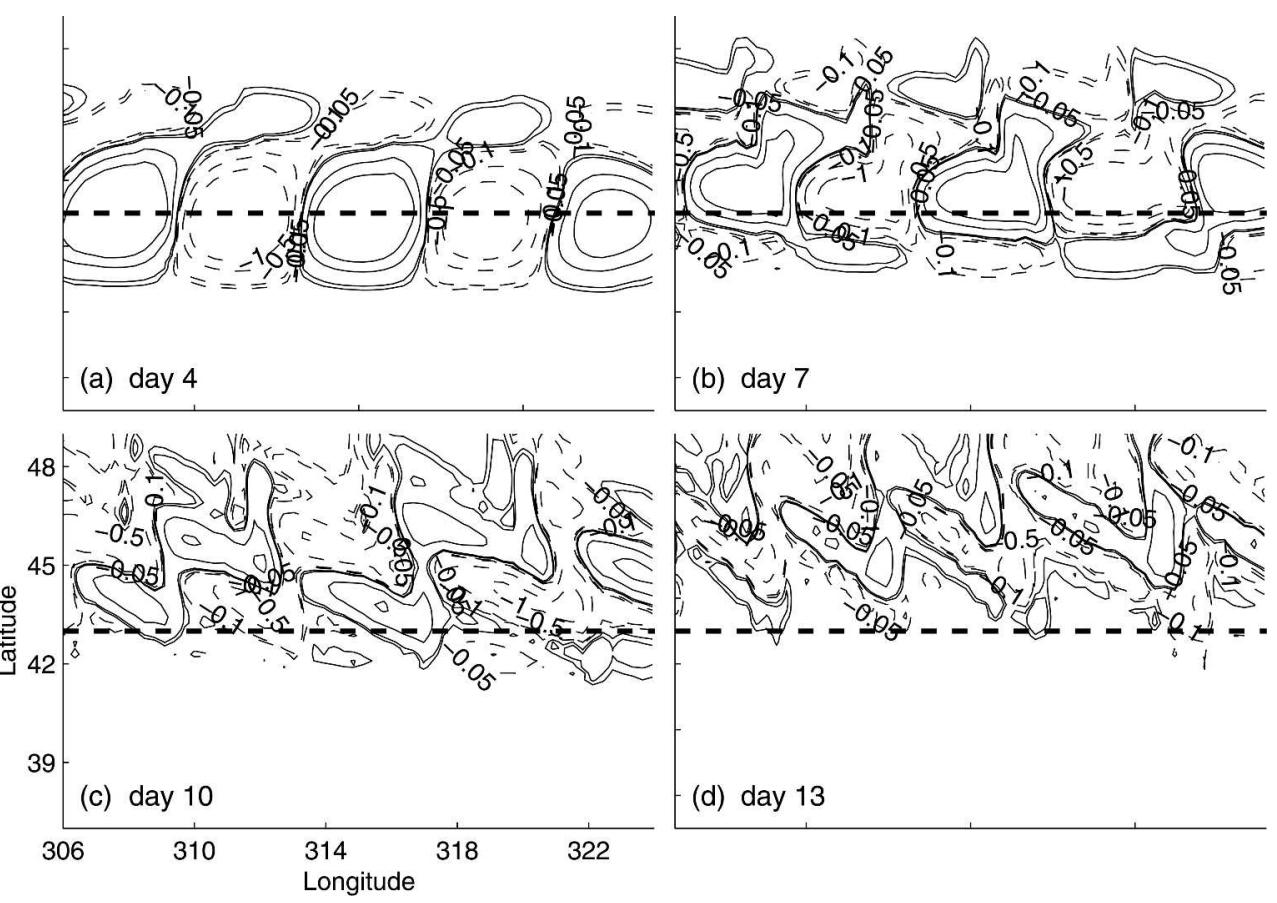

FIG. 7. Temporal evolution of the inertial-band filtered zonal current at the sea surface in the diagnostic run on a $\beta$ plane (unit: $\mathrm{m} \mathrm{s}^{-1}$ ). The dashed line represents the storm track.

of the Doppler shift effect. The inertial oscillations shrink meridionally with latitude during this advection. As the scales become smaller, the near-inertial waves are more vulnerable to nonlinear interactions, which could eventually lead to small-scale dissipation and mixing. This advection-induced mixing occurs poleward of their source regions. Since a given energy level at higher latitudes causes much more mixing than at lower latitudes (Gregg et al. 2003; Garrett 2003), a mechanism for transporting inertial energy to higher latitudes could lead to more efficient mixing than would otherwise be the case. We believe, therefore, that the mechanism described in this paper could be important for understanding mixing in the ocean. The phenomenon

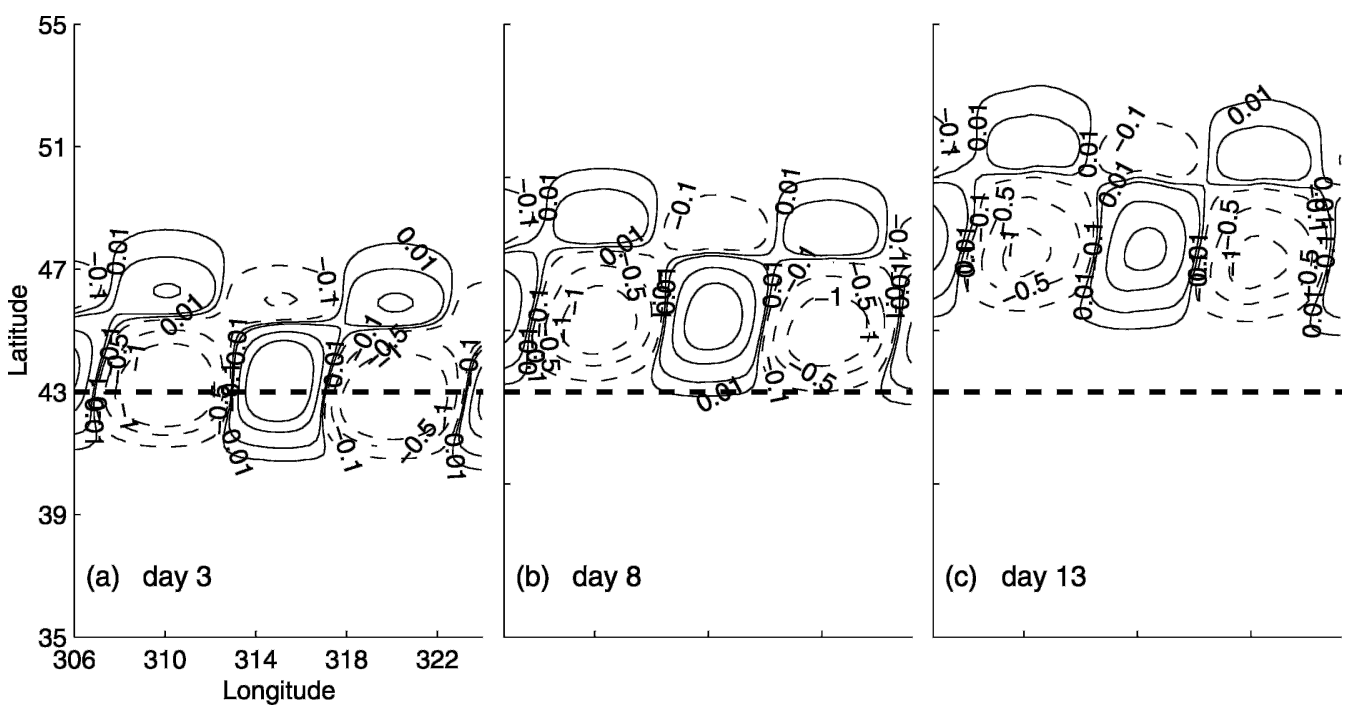

FIG. 8. Temporal evolution of the zonal current at the sea surface in the diagnostic run on an $f$ plane (unit: $\mathrm{m} \mathrm{s}^{-1}$ ). The dashed line represents the storm track. 


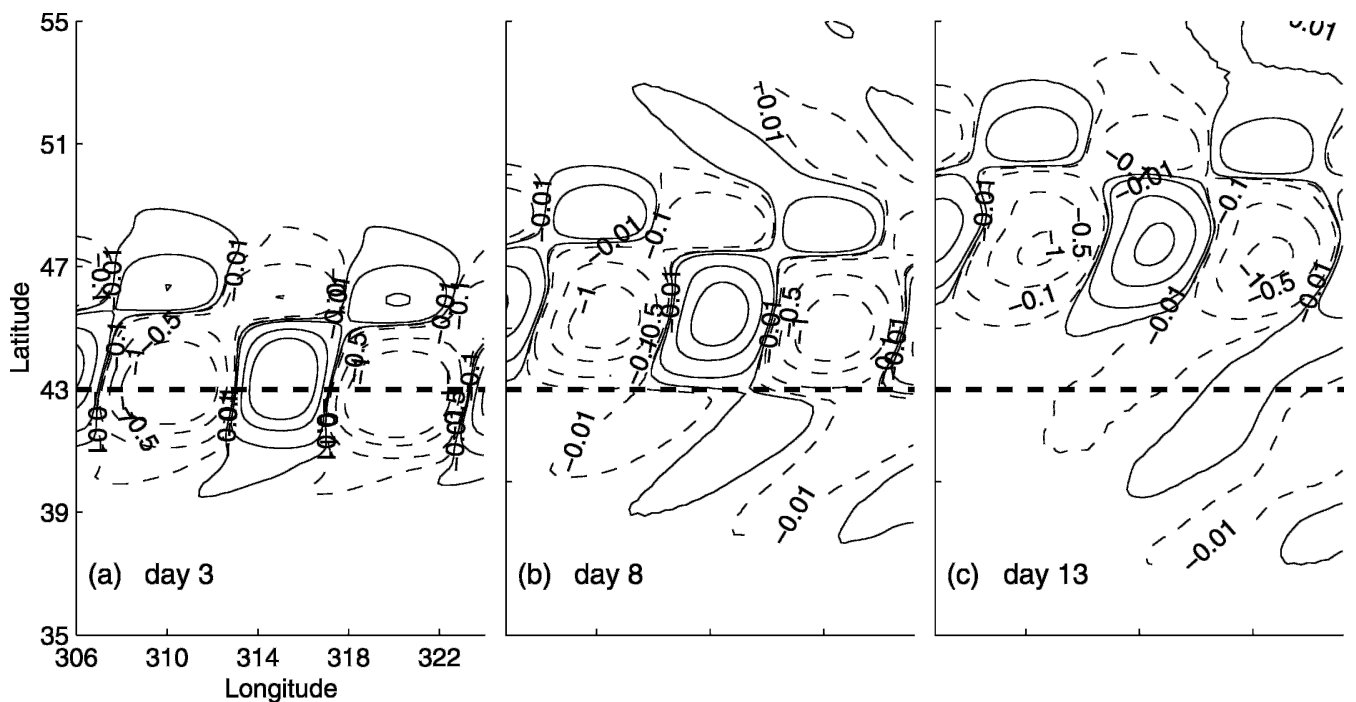

FIG. 9. Temporal evolution of the zonal current at the sea surface in the prognostic run on an $f$ plane (unit: $\mathrm{m} \mathrm{s}^{-1}$ ). The dashed line represents the storm track.

discussed in this paper could be applied to the North Atlantic Current (e.g., off eastern Canada), the Norwegian Coastal Current, and other poleward currents, even though those are more complicated environments and are subject to additional physics.

Acknowledgments. We thank Youyu Lu and Kevin Lamb for helpful discussions. This project is supported by funding from CFCAS.

\section{REFERENCES}

Alford, M. H., 2003: Redistribution of energy available for ocean mixing by long-range propagation of internal waves. Nature, 423, 159-162.

Anderson, D. L. T., and A. E. Gill, 1979: Beta dispersion of inertial waves. J. Geophys. Res., 84, 1836-1842.

Chang, S. W., and R. A. Anthes, 1978: Numerical simulations of the ocean's nonlinear baroclinic response to translating hurricanes. J. Phys. Oceanogr., 8, 468-480.

Chiswell, S. M., 2003: Deep equatorward propagation of inertial oscillations. Geophys. Res. Lett., 30, 1533-1536.

D'Asaro, E. A., 1995: Upper-ocean inertial currents forced by a strong storm. Part III: Interaction of inertial currents and mesoscale eddies. J. Phys. Oceanogr., 25, 2953-2958.

$\mathrm{Fu}$, L. L., 1981: Observations and models of inertial waves in the deep ocean. Rev. Geophys. Space Phys., 19, 141-170.

Garrett, C., 2001: What is the "near-inertial" band and why is it different from the rest of the internal wave spectrum? $J$. Phys. Oceanogr., 31, 962-971.

2003: Mixing with latitude. Nature, 422, 477-478.

Geisler, J. E., and R. E. Dickinson, 1972: The role of variable Coriolis parameter in the propagation of inertia-gravity waves during the process of geostrophic adjustment. J. Phys. Oceanogr., 2, 263-272.

Gill, A. E., 1982: Atmosphere-Ocean Dynamics. Academic Press, $662 \mathrm{pp}$.

- 1984: On the behavior of internal waves in the wake of storms. J. Phys. Oceanogr., 14, 1129-1151.

Greatbatch, R. J., 1983: On the response of the ocean to a moving storm: The nonlinear dynamics. J. Phys. Oceanogr., 13, $357-$ 367

Gregg, M. C., T. B. Sanford, and D. P. Winkel, 2003: Reduced mixing from the breaking of internal waves in equatorial waters. Nature, 422, 513-515.

Kunze, E., 1985: Near-inertial propagation in geostrophic shear. $J$. Phys. Oceanogr., 15, 544-565.

Lee, C. M., and C. C. Eriksen, 1997: Near-inertial internal wave interactions with mesoscale fronts: Observations and models. J. Geophys. Res., 102, 3237-3253.

Olbers, D. J., 1981: The propagation of internal waves in a geostrophic current. J. Phys. Oceanogr., 11, 1224-1233.

Orvik, K. A., and M. Mork, 1995: A case study of Doppler-shifted inertial oscillations in the Norwegian Coastal Current. Cont. Shelf Res., 15, 1369-1379.

Price, J. F., 1981: Upper ocean response to a hurricane. J. Phys. Oceanogr., 11, 153-175.

White, W. B., 1972: Dopper shift in the frequency of inertial waves observed in moored spectra. Deep-Sea Res., 19, 595600

Zervakis, V., and M. D. Levine, 1995: Near-inertial energy propagation from the mixed layer: Theoretical consideration. $J$. Phys. Oceanogr., 25, 2872-2889.

Zhai, X., R. J. Greatbatch, and J. Sheng, 2004: Advective spreading of storm-induced inertial oscillations in a model of the northwest Atlantic Ocean. Geophys. Res. Lett., 31, L14315, doi:10.1029/2004GL020084. 